\title{
Validez Concurrente: Escala de Inteligencia Reynolds Rías y McCarthy para la Evaluación del Desarrollo Cognitivo en Niños
}

\author{
Concurrent Validity: Reynolds Rías and McCarthy Intelligence Scale for Evaluating \\ Cognitive Development in Children
}

\author{
Miguel Alberto Montañez Romero ${ }^{1}$ y Elisama Esther Beltrán de la Rosa ${ }^{2}$
}

\begin{abstract}
Resumen
El objetivo del estudio consistió en correlacionar las Escalas de Inteligencia Reynolds Rías (RIAS) y McCarthy de aptitudes y psicomotricidad para niños (MSCA), para la evaluación del desarrollo cognitivo en niños entre 4 a 6 años, mediante un estudio transversal correlacional, con una muestra de 70 niños. Los resultados obtenidos a través de un modelo de regresión simple, confirman que entre las variables RIAS y MSCA, existe una correlación estadísticamente significativa con un coeficiente de correlación de .865 y un R cuadrado ajustado del $98.5 \%$ lo cual, señala el alto nivel de explicación del modelo, cumpliéndose, además todas las pruebas de residuos para corroborar la calidad del modelo de pronóstico. Evidenciando la validez significativa al emplear la Escala RIAS, en la evaluación del desarrollo cognitivo en los niños de 4 a 6.
\end{abstract}

Palabras clave: aptitudes, coeficiente de correlación, desarrollo cognitivo, psicomotricidad

\begin{abstract}
The objective of the study was to correlate the Reynolds Rias Intelligence Scale (RIAS) and the McCarthy Scale of Skills and Psychomotor Skills for Children (MSCA), for the evaluation of cognitive development in children between 4 and 6 years of age, by means of a cross-sectional correlational study, with a sample of 70 children. The results obtained through a simple regression model confirm that there is a statistically significant correlation between the RIAS and MSCA variables, with a correlation coefficient of .865 and an adjusted R-squared of $98.5 \%$, which indicates the high level of explanation of the model, also fulfilling all the residual tests to corroborate the quality of the prognostic model. Evidencing the significant validity of using the RIAS Scale in the evaluation of cognitive development in children from 4 to 6 years old.
\end{abstract}

Keywords: aptitudes, correlation coefficient, cognitive development, psychomotor skills

\footnotetext{
${ }^{1}$ Psicólogo, Magister en Psiconeuropsiquiatría y Rehabilitación Universidad Metropolitana, Candidato a Doctor en Neurociencia Cognitiva Aplicada Universidad de la Costa, Docente de la Universidad Metropolitana de Barranquilla. Investigador del grupo CEPUM, Universidad Metropolitana, Colombia. Orcid.org/0000-0002-4534-4515. E-mail: miguel.montañez@unimetro.edu.co; miguelmontanez1989@gmail.com ${ }^{2}$ Doctora en Psicología Comportamiento y Cognición, Altlantic International University- EE.UU. Magíster en Psiconeuropsiquiatría y Rehabilitación, Universidad Metropolitana. Magíster en Psicología en Intervención Familiar Columbus IBS-Aragón, España. Especialista en Salud Familiar Universidad del Norte, Especialista en VIH, Asociación Colombiana de Infectología. Especialista en Psicología en Intervención Familiar Columbus IBS-Aragón, España. Director Programa de Psicología, Universidad Metropolitana, Barranquilla. Docente investigador, grupo CEPUM, Universidad Metropolitana. CP 0008001. Correo: elisama.beltran@unimetro.edu.co; elisamabeltran@gmail.com. Código Orcid: https://orcid.org/0000-0002-3119-2944
} 


\section{Introducción}

Abordar el tema de la evaluación neurocognitiva en niños se ha convertido en un constructo de importancia para la psicología y la neuropsicología, precisando dar una mirada al desarrollo cognitivo, psicoafectivo, escolar y social, entre otros; ámbitos que demandan ser evaluados, por motivos específicos, comprometiendo al profesional en la búsqueda de instrumentos, con características específicas que satisfagan la necesidad. Por tal razón, estas pruebas, deben poseer una validez que permita proporcionar información relevante en el alcance de un diagnóstico confiable y/o diferencial, al momento de determinar el nivel de alteración que se presente, conduciendo a estimar resultados funcionales relevantes en la planeación de la intervención, constituyéndose como punto de partida para establecer el éxito del tratamiento a futuro (Aragón, 2004; Eloi 2019) y pronóstico de la evolución final del mismo (Comisión Nacional de Protección Social en Salud 2013).

Por lo tanto, es importante considerar, que la evaluación neurocognitiva no se deriva directamente de un instrumento de evaluación de área específico. Sino que cualquier test con fundamentación teórico conceptual y con aspectos relacionados como: fiabilidad, validez $\mathrm{y}$ sensibilidad a los cambios clínicos, viabilidad de administración, la validación de las pruebas con poblaciones similares, la aplicación de las mismas por diferentes grupos de trabajo, protocolos claros y precisos de administración y puntuación, puede ser utilizado con éxito dentro del marco de una evaluación neurocognitiva. Como es descrito por Benedet, (2002) no solo un test, hace todo por sí mismo, para lograrlo, es preciso utilizar un conjunto de test complementarios, establecidos de antemano que permitan una exploración mucho más amplia del niño en su desarrollo evolutivo.

No obstante, un aspecto que se debe señalar e importante dentro del proceso de evaluación del desarrollo cognitivo, es la edad, al momento de la evaluación. En lo que se respecta a niños en edades entre 4 a 6 años, se debe considerar que las adquisiciones evolutivas son relevantes en el desarrollo del lenguaje, motricidad, control de esfínter, autonomía, y primeros aprendizajes escolares. De igual manera, los cambios cuantitativos y cualitativos son sorprendentes, y, quizás por ello, los desfases madurativos pueden considerarse esperables o dentro de lo normal. Razón por la cual, los instrumentos psicométricos a utilizarse, estarán encaminados a la evaluación del desarrollo cognitivo del niño y la capacidad madurativa mental. En general, para la evaluación del niño en edad preescolar se requieren de actividades variadas y de corta duración, que demanden la participación activa y motivación de parte de ellos, en miras a obtener unos resultados pertinentes y coherentes (Santana, et al., 2006).

Por lo cual, estos procesos requieren de un esfuerzo continuo para hallar medidas de detección con las condiciones que se exigen en una prueba tales como, la sensibilidad y especificidad (Balsamo et al., 2019). Sin embargo, es importante hallar pruebas concurrentes, enfocadas en evaluar y diagnosticar diferentes funciones en un solo espacio, asimismo, pueden ser aprovechadas para monitorear el avance en el nivel de aprendizaje a lo largo del tiempo, (Covacevich, 2014). Facilitando a quienes evalúan menor inversión del tiempo, con la misma confiabilidad y pertinencia. Así mismo, la evaluación sistemática y masiva del desarrollo infantil temprano es una eficaz forma de prevención de los trastornos del desarrollo (Leal et al., 2005) y, sobre todo, las de orden técnico: la mayor eficacia, eficiencia y simplicidad de las medidas tempranas por sobre aquellas tomadas más tardíamente (Lira, 1992).

Con estos fines de evaluación dentro de la oferta actual de pruebas para evaluación del desarrollo cognitivo como la escala McCarthy de aptitudes y psicomotricidad (MSCA) y la escala de inteligencia de Reynolds (RIAS), es importante, identificar los criterios de cada una en estructura y componentes.

La escala de MSCA, permite evaluar el desarrollo cognitivo y motriz del niño, a través de una batería conformadas por 5 escalas (Verbal, Perceptivo-Manipulativa, Numérica, General, Cognitivo, Memoria y Motricidad) y 18 sub-test, proyectada para su aplicación en niños de edades entre $3-8$ años. Prueba que requiere en niños menores de 5 años de 45 - 50 minutos y de niños 5 años alrededor de 1 hora.

La confiabilidad de las escalas MSCA se encuentra estimada mediante el cálculo de los 
coeficientes de consistencia interna (dos mitades con la corrección de Spearman-Brown) y estabilidad, así como el cálculo de los errores típicos de medida. En la escala general cognitiva el coeficiente promedio de fiabilidad por el procedimiento de dos mitades para los grupos de edad fue de .93. Los promedios en las demás escalas varían de .79 a .88 . Los coeficientes de fiabilidad también reflejan un alto grado de estabilidad, con un valor aproximado de .90 en la escala GC y correlaciones que variaban de 0.75 y .89 en las demás escalas. Siguiendo el mismo orden de ideas, los estudios de fiabilidad alcanzan valores entre .90 y .96 para el IGC (índice general cognitivo) y entre .60 y .62 para el resto de las escalas. Se han efectuado distintos estudios de validación, alcanzándose resultados variados en general y aceptable. Acerca de la escala de inteligencia RIAS, como test de inteligencia de aplicación individual indicado para evaluar sujetos desde los 3 y 94 años de edad, proporciona un índice de inteligencia verbal (IV), a partir de los resultados de sus dos sub-escalas (adivinanza y analogías verbales) un índice de inteligencia no verbal (INV), a partir de los resultados de otras dos (Categorías y Figuras incompletas). La suma transformada de las puntuaciones $\mathrm{T}$ de las cuatro pruebas da como resultado el índice de Inteligencia general (IG), (IV+INV=IG) siendo una estimación sintética de la inteligencia global. Por lo tanto, la aplicación de estas cuatro pruebas de inteligencia por parte de un examinador entrenado y experto requiere de 25 a 35 minutos aproximadamente. El índice de Memoria general (IM) se calcula a partir de las dos pruebas complementarias de memoria, cuya aplicación requiere aproximadamente 10 minutos adicionales. La palabra general en los índices de Inteligencia general y Memoria general indica que ambos índices representan la combinación de pruebas verbales y no verbales. Los índices compuestos (IG e IM) se distinguen de los índices verbal y no verbal en que estos dos últimos representan un único factor, es decir, verbales y no verbal (Reynolds et al., 2009).

De acuerdo a las condiciones de criterio la escalas de RIAS, en relación a la fiabilidad, la consistencia interna de los elementos de las pruebas por edades, se obtuvo mediante la aplicación de una Alfa de Cronbach (Cronbach,
1951). Obteniendo por resultados medianas de .89 para Adivinanzas, .86 para Categorías, .89 para Analogías verbales, .82 para Figuras incompletas, de igual manera para la consistencia interna de los índices del RIAS se obtuvo a través de la fórmula de Guilford. (Guilford, 1954) con resultados de: .93 para IV, .90 para INV y .94 para IG. Los estudios de fiabilidad alcanzan valores entre .90 y .96 para el IGC (índice general cognitivo) y entre .60 y .62 para el resto de las escalas. Se han efectuado distintos estudios de validación, alcanzándose resultados variados, en general aceptables.

Es importante destacar, que la Escala MSCA de Aptitudes y Psicomotricidad para Niños evalúa el desarrollo cognitivo del niño teniendo en cuenta, que los sustratos que los conforman son las habilidades verbales, no verbales, visomotoras, memoria, funciones ejecutivas. En comparación la escala de RIAS, permite, hacer una evaluación de la inteligencia, sin la interferencia de procesos viso-motores $y$ velocidad de procesamiento. Incluso es una prueba que puede ser aplicada a niños con cierta discapacidad física sin que se afecten los resultados esperados de coeficiente intelectual.

Ahora bien, partiendo de las propiedades psicométricas ya establecidas y verificadas del MSCA, se tomará a esta prueba como criterio de validez concurrente para el RIAS, en cuanto al índice global de inteligencia que este arroja, teniendo en cuenta que el objetivo del RIAS, con sus sub pruebas es la identificación de un coeficiente intelectual, se esperaría que los resultados del mismo mantuvieran una congruencia con aspectos del desarrollo cognitivo previamente encontradas como correlación entre el CGI y la MSCA.

Por lo tanto, el objetivo del presente estudio es correlacionar los resultados obtenidos en la evaluación cognitiva infantil entre la escala MSCA y la RIAS, para corroborar la validez en la evaluación del desarrollo cognitivo de la RIAS en niños. Facilitando de esta forma una evaluación desde estas perspectivas.

\section{Método}

Para el presente estudio, se seleccionan 70 niños, con edades comprendida entre los cuatro a seis años adscritos al Servicio de Psicometría y 
Tabla 1. Estructura y componentes de escala MSCA y la RIAS

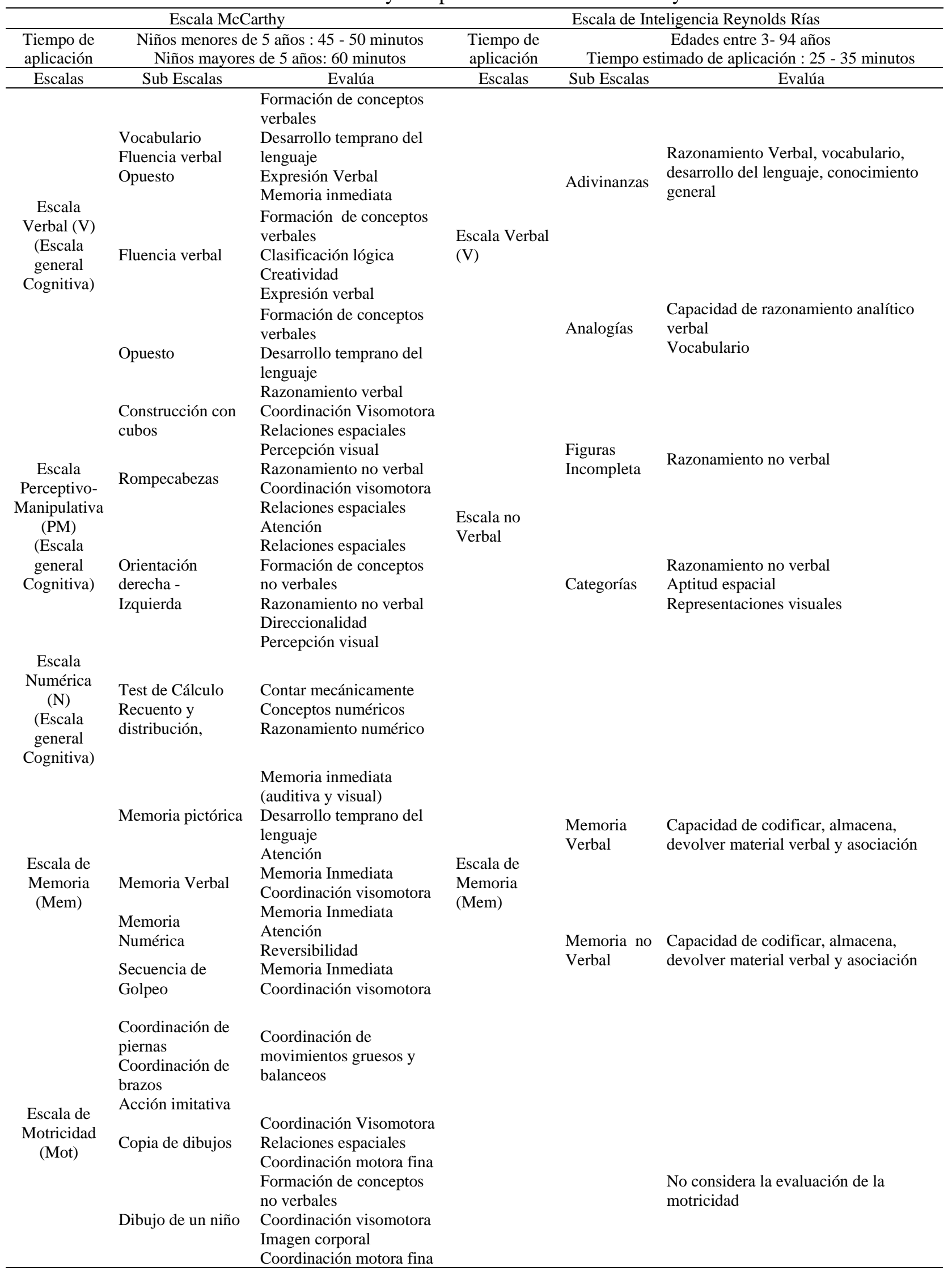

Fuente: construcción propia, basada en ambas escalas

Rehabilitación Mental, de la Fundación Hospital
Universitario Metropolitano (FHUM) en la ciudad
de Barranquilla, Colombia. Esta investigación se circunscribe a un estudio transversal correlacional, involucrando variables demográficas tales como edad, sexo y otras variables derivadas de las 
escalas: MSCA, tales como: escala verbal, escala perceptivo- manipulativa, escala numérica, índice global general (GCI) y la escala RÍAS: Índice verbal, Índice no verbal e índice global.

Para el desarrollo del estudio se consideró aplicar las escalas de McCarthy. MSCA y la de inteligencia Reynolds RIAS, ambas con sus respectivas sub-escalas necesaria para la obtención del Cociente General de Inteligencia (CGI) primero la MSCA y posteriormente, con un día de diferencia se aplicó la escala RIAS, con sus sub escalas necesarias para la obtención del índice Global (IG).

Las pruebas fueron aplicadas por profesionales expertos en el área, con ambientes controlados: buena iluminación y libres de ruido. El tiempo de aplicación aproximado fue de 60 minutos para la prueba de MSCA y 45 minutos para las siete sub-pruebas del RIAS.

En relación con el objetivo del estudio planteado acerca de correlacionar las Escalas RIAS y la MSCA, se realiza una comparación de la estructura y componente de cada una de las escalas (Tabla 1).

El análisis estadístico, se desarrolló mediante un modelo de regresión simple y se calculó el coeficiente de correlación de Pearson. Para establecer la medición del nivel de concordancia entre estas variables: variable dependiente (MSCA) y variable Independiente (RIAS) se analizó el $\mathrm{R}$ cuadrado ajustado del modelo de regresión, así como el valor el Coeficiente de Correlación de Pearson, además de corroborar la capacidad del modelo, para explicar el comportamiento del MSCA se realizó análisis de los residuos del modelo (análisis gráfico y la prueba de normalidad).

\section{Resultados}

Según los hallazgos obtenidos, se evidencia una edad media de 5 años y una desviación estándar de .62. Los resultados obtenidos, entre los promedios generales, entre las escalas MSCA y RIAS, en relación al Cociente General de Inteligencia (CGI), y el Índice global (IG), muestran una diferencia de 1.18 entre ellas, siendo poco amplia, permitiendo establecer una relación significativa entre ambas como se muestra en la Tabla 2 (parte B).
Tabla 2. Variables demográficas

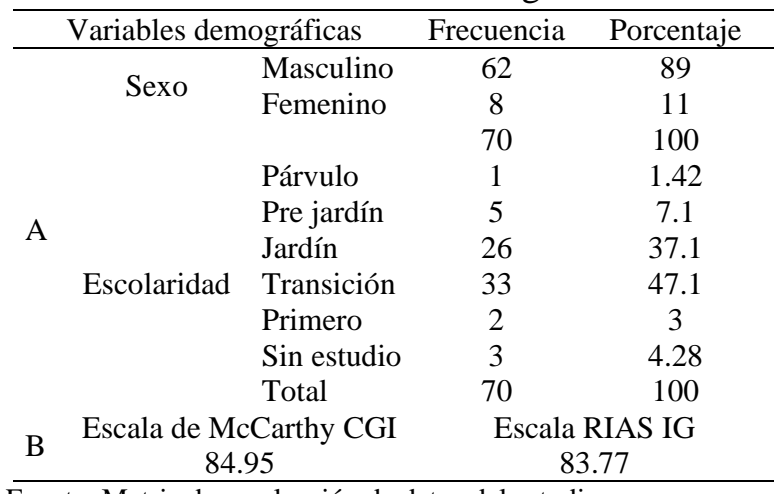

Por otro lado, al realizar la validez concurrente entre las variables: Variable dependiente: (MSCA) y Variable independiente: Escala RIAS, se comprueba a través del Coeficiente de Correlación de Pearson, un índice de 865 el cual indica una fuerte correlación entre las dos escalas, como se puede ver en la Tabla 3.

Tabla 3. Correlación de Pearson en relación a MSCA y a RIAS

\begin{tabular}{clcc}
\hline & & CGI & IG \\
\hline \multirow{4}{*}{ MSCA } & Correlación de & 1 & $0.865^{* *}$ \\
& Pearson & & 0 \\
& Sig. (bilateral) & 70 & 70 \\
& N & $.865^{* *}$ & 1 \\
& Correlación de & 0 & \\
\multirow{2}{*}{ RIAS } & Pearson & 70 & 70 \\
& Sig. (bilateral) & & \\
& N & &
\end{tabular}

Fuente: Análisis estadístico SSPS

Los resultados ajustados a un modelo lineal permitieron describir la relación entre el CGI y IG obteniéndose una ecuación del modelo ajustado para CGI (MSCA) $=1.01 *$ IG, (RIAS), ello, permite explicar que todo resultado de un índice RIAS, arrojará una puntuación lo suficientemente confiable para correlacionar con el CGI del MSCA. Dicho hallazgo se puede comprobar en que el valor-p en la tabla ANOVA es menor que 0.05 , lo cual muestra que existe una relación estadísticamente significativa entre CGI (MSCA) e índice global (IG) con un nivel de confianza del 95\% (Tabla 4).

Tabla 4. Coeficientes modelo Lineal

\begin{tabular}{ccccc}
\hline & $\begin{array}{c}\text { Mínimos } \\
\text { Cuadrados }\end{array}$ & Estándar & Estadístico & \\
\hline Parámetro & Estimado & Error & $\mathrm{T}$ & Valor-P \\
Pendiente & 1.01 & 0.01 & 67.57 & 0 \\
\hline Fuente: Análisis estadístico SSPS & &
\end{tabular}

Fuente: Análisis estadístico SSPS 
El estadístico R-Cuadrada indica que el modelo ajustado explica $98.5 \%$ de la variabilidad en CGI (MSCA). El coeficiente de correlación es igual a .99 indicando una relación relativamente fuerte entre las variables. El error estándar del estimado indica que la desviación estándar de los residuos es 10.75, este valor puede usarse para construir límites de predicción para nuevas observaciones (Tabla 5).

Tabla 5. Estadístico R-cuadrada

\begin{tabular}{lc}
\hline Coeficiente de Correlación & 0.99 \\
R-cuadrada & $98.5 \%$ \\
R-cuadrado (ajustado para g.1.) & $98.5 \%$ \\
Error estándar del est. & 10.75 \\
\hline Fuente: Análisis estadístico SSPS &
\end{tabular}

Sin embargo, para evaluar si el modelo es acorde para explicar el comportamiento del MSCA e incluso pronosticar valores del mismo, se aplicaron las pruebas de comportamiento a los residuos o errores de pronóstico del modelo, donde se demostró que los errores de pronóstico no guardan ningún patrón de comportamiento con respecto a la variable independiente, número de fila ni con respecto a los valores predichos (Tabla 6).

Tabla 6. Pruebas de Bondad-de-Ajuste para RESIDUOS

\begin{tabular}{lc}
\hline & Normal \\
\hline DMAS & 0.05 \\
DMENOS & 0.07 \\
DN & 0.07 \\
Valor-P & 0.78 \\
\hline
\end{tabular}

Fuente: Análisis estadístico SSPS

Por último, se realizó una comparación descriptiva de los porcentajes según los niveles de interpretación de los resultados finales de cada una de las pruebas tanto del MSCA como del RIAS, obteniendo que no existen mayores diferencias en la distribución de la muestra teniendo en cuenta los resultados finales obtenidos tanto en el CGI como el IG, respectivamente, solo a nivel del medio alto donde los porcentajes presentan una diferencia superior a 5 puntos (Tabla 7).

Tabla 7. Distribución de porcentajes según nivel de compromiso final

\begin{tabular}{lcc}
\hline Prueba & Mccarthy & Rias \\
\hline Nivel de Compromiso & Porcentaje & Porcentaje \\
\hline Muy Inferior & 19 & 17 \\
Inferior & 14 & 20 \\
Medio Bajo & 21 & 19 \\
Medio & 34 & 39 \\
Medio Alto & 12 & 4 \\
Superior & 0 & 1 \\
\hline
\end{tabular}

Fuente: Propia

\section{Discusión}

Existen estudios, sobre la validez de distintos instrumentos, tal es el sistema del dibujo de la figura humana (DFH) que evalúan la capacidad intelectual, al efectuar un índice de correlación significativo con el WISC los resultados del estudio realizado muestran un índice de correlación significativos con valores variables, predominando los bajos y moderados. La revisión de Harris (1991) sobre el Test del Dibujo de un Hombre de Goodenough (1926) mostró correlaciones significativas con el cociente intelectual total (CIT) del WISC que se situaban entre .13 y .70. En la revisión de Scott (1981) sobre las publicaciones del sistema Goodenough (Harris, 1991) se mencionan estudios que encontraron correlaciones significativas moderadas y altas entre la escala de puntos de este sistema y los CI del WISC-R (Fernández et al., 2017).

Mediante estos antecedentes descritos, los resultados obtenidos, sugieren la validez concurrente entre la prueba de MSCA, y la escala RIAS. Evidenciando así que las relaciones encontradas sugieren, en general que la escala de RIAS, cuenta con validez concurrente, correlacionada con la escala de MSCA, así, como una congruencia con la estructura teórica de Cattel, Horn y Carroll (1966). Es decir, puede ser aplicada para estimar de manera aceptable un Índice de Desarrollo Global, como el expresado por la escala CGI del MCSA, descartando la influencia de los procesos viso construccionales y práxicos dentro de la evaluación del desarrollo cognitivo, como si se obtiene con la anterior escala mencionada.

En cuanto los resultados categorizados por niveles de afectación muestran, por ejemplo: en un nivel muy inferior en el CGI del $19 \%$ y un IG del $17 \%$, lo que evidencia una diferencia mínima de 2 puntos entre ambos resultados. Con respecto al nivel inferior los resultados para el CGI fueron del $14 \%$ y $20 \%$ en el IG, lo cual muestra una diferencia de 4 puntos porcentuales. En el mismo orden de ideas, al nivel medio bajo la diferencia porcentual es de 2 puntos entre los resultados del CGI con un $21 \%$ de la muestra contra un 19\% de la misma muestra en este nivel. Por ultimo en cuanto al nivel de desempeño medio los 
resultados igualmente muestran una consistencia en su relación, en tanto la diferencia porcentual es de 5 puntos ya que los resultados del CGI en este rango fueron del $34 \%$ de la muestra, mientras que para el IG fue del $39 \%$. Estos resultados evidencian que existe una congruencia entre ambas pruebas al momento de categorizar según los niveles obtenidos en sus respectivos resultados.

Así de esta forma, se convierte el RIAS en un instrumento fiable y válido para la exploración y evaluación clínica del desarrollo cognitivo en los niños de 4 a 6 años y el CGI, evidenciado a través de la prueba estadística de Correlación de Pearson, demostrado con una $p=.865$, evidenciando una correlación positiva considerable entre ambas pruebas.

Ahora bien, con este resultado podemos concluir que existe una correlación entre las dos pruebas. Permitiendo establecer que, al utilizar la escala RIAS podemos obtener unos resultados confiables para la evaluación del desarrollo cognitivo en los niños de 4 a 6 años.

\section{Consideraciones éticas}

Dentro de las consideraciones éticas se tuvo en cuenta la aplicación del consentimiento informado y el aval del comité de Bioética de Investigación de la Universidad Metropolitana y del FHUM, de la ciudad de Barranquilla instituciones que apoyaron la investigación.

\section{Conflicto de intereses}

Sin conflicto de interesses.

\section{Referencias}

Aragón, L. (2004). Fundamentos psicométricos en la evaluación psicológica. Revista de Psicología Iztacala, 7(4), 23-43. Recuperado de http://www.revistas.unam.mx/index.php/repi/a rticle/view/21668

Balsamo, L., Mitcherll, H., Ross, W., Metayer, C., Hardy,..\& K., Kadan. (2019). Monitoring neurocognitive functioning in childhood cancer survivors: Evaluation of CogState computerized assessment and the Behavior Rating Inventory of Executive Function (BRIEF). BMC Psychology Journal, 7(1), 1-8. doi.org10.1186/s40359-019-0302-3.

Benedet, M. (2002). Neuropsicología Cognitiva. Aplicaciones a la clínica y a la investigación Fundamento teórico y metodológico de la Neuropsicología Cognitiva. Madrid, España: Documento Técnico $\mathrm{N}^{\circ} 1$. Observatorio de la Discapacidad. ODC. Recuperado de: https://www.imserso.es/InterPresent2/groups/i mserso/documents/binario/neuropsicologia.pdf

Comisión Nacional de Protección Social en Salud (2013). Manual para la evaluación de menores de cinco años con riesgo de retraso en el desarrollo. $\left(1^{\circ}\right.$ ed.) México D.F: Secretaría de Salud. Recuperado de: http://himfg.com.mx/descargas/documentos/E DI/ManualparaEvaluacionaMenoresde 5conRi esgodeRetrasoenelDesarrollo.pdf

Covacevich, C. (2014). Cómo seleccionar un instrumento para evaluar aprendizajes estudiantiles. Banco Interamericano de Desarrollo BID. Nota Técnica del BID; 738 Pág. 4. Recuperado de: https://publications.iadb.org/publications/span ish/document/C\%C3\%B3mo-seleccionar-uninstrumento-para-evaluar-aprendizajesestudiantiles.pdf

Cronbach, L. (1951). Coefficient alpha and the internal structure of test. Psychometrika Journal, 16(3), 297-334.

McGrath, N., \& Eloi, J. (2019). The role of neuropsychology in the evaluation of concussion. Seminars in Pediatric Neurology, 30, 83-95. doi.org/10.1016/j.spen.2019.03.013

Nistal, M. T. F., Zaragoza, F. A. P., \& Ávila, E. O. (2018). La validez del dibujo de la figura humana como una medida de la madurez intelectual en niños indígenas Yaquis. Revista Iberoamericana de Diagnóstico y Evaluación - e Avaliação Psicológica, 3(48), 41-52. doi.org/10.21865/RIDEP48.3.04

Guilford, J. P. (1954). Psychometric methods. $\left(2^{\circ}\right.$ ed.) Editorial McGraw-Hill.

Horn, J., \& Cattel, R., (1966). Refinerment and test of the of the theory of fluid and crystallized general intelligences. Journal of Educational Psychology, 57(5), 253-270. doi.org/10.1037/h0023816

Leal, F., Daudet A., Donaire, D., \& Segovia, M. (2005). Un instrumento para la evaluación desarrollo infantil con participación de los 
padres. Revista Iberoamericana de Diagnóstico y Evaluación - e Avaliação Psicológica, 19(1); 55-77. Recuperado: http://www.redalyc.org/articulo.oa?id=459645 450004

Lira, L., \& Isabel, M. (1992). Construcción y evaluación de una técnica de rastreo de retrasos del desarrollo psicomotor: Segundo año de vida. Revista Chilena de Pediatría, 159-165. Recuperado de https://pesquisa.bvsalud.org/portal/resource/pt /lil-112455

McCarthy, D. (1988). MSCA. Escalas McCarthy de Aptitudes y Psicomotricidad para Niños (Manual) ( $3^{\circ}$ ed.). Editorial Madrid TEA.

Reynolds, C., Kamphaus, R., Fernández, P., \& Pinto I. (2009). RIAS: Escalas de inteligencia de Reynolds y RIST: test de inteligencia breve de Reynolds. Editorial TEA.

Reynolds, C., Kamphaus, R. (2013). RIAS, Escalas de Inteligencia de Reynolds. Editorial TEA.

Santana, A. C., Rojas, M., \& Pacheco, J. S. (2006). Escalas McCarthy de Aptitudes y Psicomotricidad para Niños. Revista Avances en Medición, .4(1), 148-149. Recuperado de https://www.humanas.unal.edu.co/lab_psicom etria/application/files/2616/0463/3541/Vol_4. _Resea_Escalas_McCarthy_De_Aptitudes_ Y_Psicomotricidad_Para_Nios.pdf

Tirapu, J. (2007). La evaluación neuropsicológica. Journal Psychosocial Intervention, 16(2), 189-211. 